Geological Society, London, Special Publications

\title{
Slip-system and EBSD analysis on compressively deformed fine-grained polycrystalline olivine
}

R. J. M. Farla, J. D. Fitz Gerald, H. Kokkonen, A. Halfpenny, U. H. Faul and I. Jackson

Geological Society, London, Special Publications 2011, v.360; p225-235.

doi: $10.1144 /$ SP360.13

$\begin{array}{ll}\begin{array}{l}\text { Email alerting } \\ \text { service }\end{array} & \begin{array}{l}\text { click here to receive free e-mail alerts when } \\ \text { new articles cite this article }\end{array} \\ \begin{array}{l}\text { Permission } \\ \text { request }\end{array} & \begin{array}{l}\text { click here to seek permission to re-use all or } \\ \text { part of this article }\end{array} \\ \text { Subscribe } & \begin{array}{l}\text { click here to subscribe to Geological Society, } \\ \text { London, Special Publications or the Lyell } \\ \text { Collection }\end{array}\end{array}$

Notes

(C) The Geological Society of London 2012 


\title{
Slip-system and EBSD analysis on compressively deformed fine-grained polycrystalline olivine
}

\author{
R. J. M. FARLA ${ }^{1}$, J. D. FITZ GERALD ${ }^{1 *}$, H. KOKKONEN ${ }^{1}$, A. HALFPENNY ${ }^{1}$, \\ U. H. FAUL ${ }^{2} \&$ I. JACKSON ${ }^{1}$ \\ ${ }^{1}$ Research School of Earth Sciences, Building 61, The Australian National University, \\ Canberra ACT 0200, Australia \\ ${ }^{2}$ Department of Earth Sciences, Boston University, 675 Commonwealth Ave., Boston, \\ MA 002215, USA \\ *Corresponding author (e-mail: john.fitzgerald@anu.edu.au)
}

\begin{abstract}
A slip-system analysis was performed on two synthetic compressively deformed olivine aggregates, derived from experimental solution-gelation (sol-gel) and natural San Carlos precursors to determine how dislocation density relates to Schmid factor for slip in olivine. Individual grain orientations were measured with electron backscatter diffraction. Using decorated dislocations, grain populations were separated into subsets of high versus low dislocation density. Analysis of preferred orientations and distributions of Schmid factors suggests that there is only weak correlation between Schmid factor and dislocation density, slip on (010)[100] in San Carlos grains but (001)[100] in sol-gel material, with multiple slip or stress heterogeneity in both.
\end{abstract}

Olivine, the main constituent of the Earth's upper mantle, has been thoroughly studied in the past decades to understand how crystallographic preferred orientations (CPO) develop under upper mantle conditions due to prevailing plate tectonic forces (Ben Ismaïl \& Mainprice 1998; Tommasi et al. 2000; Jung et al. 2006; Karato 2008), but less attention has been given to fine-grained polycrystalline olivine. CPO development commonly occurs in coarse-grained olivine during plastic deformation (i.e. high-temperature dislocation creep, $>1000{ }^{\circ} \mathrm{C}$ ) where individual grains rotate and/or recrystallize, promoting anisotropy in seismic measurements (Karato 1988; Lee et al. 2002; Drury \& Pennock 2007). For a fine-grained aggregate, however, stabilization of grain size below that at which extensive subgrain development takes place decreases the probability of recrystallization (White 1979) and is observed in essentially dry, solution-gelation (sol-gel) derived olivine (Faul et al. 2011) or ultra-mylonites in shear zones.

Several slip systems potentially operate in olivine during plastic deformation. According to the von Mises criterion, five independent slip systems are needed for maximum strain compatibility (Von Mises 1928). For each slip system (hkl)[uvw], a geometrical Schmid factor $S$ provides a measure of resolved shear stress $(0 \leq$ value $\leq 0.5)$ from the crystal orientation relative to external stress (Hull $\&$ Bacon 2001). For uniaxial compression, the Schmid factor is defined

$$
S=\cos a \times \cos b
$$

where $a$ is the angle between compression direction and slip plane normal and $b$ is the angle between compression direction and slip direction. For a polycrystalline aggregate, homogeneous stress must be assumed to calculate $S$ for each grain.

Studies on the fabric and seismic anisotropy of olivine have shown that dominant slip systems relate to the critical resolved shear stress on each slip system and temperature. Other dependencies can include chemical potential of components such as oxygen and silica (in the case of olivine) and 'water'.

The type-A CPO typically develops in low-OH natural olivine for high-temperature plastic deformation (Green \& Radcliffe 1972; Goetze 1978; Ben Ismail \& Mainprice 1998; Jung \& Karato 2001). This CPO is characterized in shear deformation by [100] grain directions subparallel to the shear direction and [010] subnormal to the shear plane. Models employing the three most active slip systems in olivine, (010)[100], (001)[100] and (010)[001] (Ribe \& Yu 1991; Wenk et al. 1991), can replicate this CPO type. Additional slip systems may also be operating, for example, $(0 k l)$ [100] (Passchier \& Trouw 1998). The range of observed CPO types is summarized in Jung et al. (2006) for a variety of water concentrations in olivine.

Two end-member states describe the interactions between grains during deformation, the uniform strain (Taylor) and uniform stress (Sachs) models (e.g. Winther et al. 1997; Dawson \& Wenk 2000). In the Taylor model, some grains require higher 
Table 1. Rheological and microstructural data of sol-gel and San Carlos samples deformed at $1250{ }^{\circ} \mathrm{C}$

\begin{tabular}{lcccccrr}
\hline Sample & $\begin{array}{c}\text { Strain, } \\
\varepsilon_{\text {total }}(\%)\end{array}$ & $\begin{array}{c}\text { Differential stress, } \\
\sigma_{\max }(\mathrm{MPa})\end{array}$ & $\begin{array}{c}\text { Strain rate, } \\
\varepsilon_{\max } / \Delta t\left(\mathrm{~s}^{-1}\right)\end{array}$ & $\begin{array}{c}\text { Grain size, } \\
d(\mu \mathrm{m})\end{array}$ & $\begin{array}{c}\rho_{\text {low }} \\
\left(\mu \mathrm{m}^{-2}\right)\end{array}$ & $\begin{array}{c}\rho_{\text {high }} \\
\left(\mu \mathrm{m}^{-2}\right)\end{array}$ & $\begin{array}{r}\rho_{\text {mean }} \\
\left(\mu \mathrm{m}^{-2}\right)\end{array}$ \\
\hline Sol-gel & 18.9 & 266 & $1.1 \times 10^{-4}$ & $4.2 \pm 0.2$ & c. 1 & c. 15 & $8.5 \pm 0.2$ \\
San Carlos & 15.0 & 227 & $1.0 \times 10^{-4}$ & $13.0 \pm 0.5$ & c. 2 & c. 16 & $11.4 \pm 2.9$ \\
\hline
\end{tabular}

grain-boundary stresses than others to produce uniform strain. In the Sachs model, some grains in favourable orientations deform more than neighbouring grains; some unfavourably oriented grains do not deform at all. In general, a positive correlation between dislocation density $(\rho)$ and Schmid factor is more likely if grain interactions obey the Sachs model rather than the Taylor model (Karato \& Lee 1999). However, it is likely that the deformation involves heterogeneous stress or strain as implemented in the Taylor-Bishop-Hill model (Lister et al. 1978) and the self-consistent model for a limited number of slip systems (Wenk et al. 1991; Tommasi et al. 2000). More recent modelling requires activation of four essential slip systems in olivine plus another degree of freedom to describe the microscopic stress heterogeneities (Castelnau et al. 2008).

This study builds upon previous studies by Karato \& Lee (1999) and Lee et al. (2002) and investigates to what extent the Taylor and Sachs models are applicable in deformed polycrystalline olivine by comparing fine-grained sol-gel olivine with a coarser-grained San Carlos for high-stress (but low-strain) compressive deformation. A robust analysis of slip systems from large populations of grains with high- and low-dislocationdensities has been possible because grain rotation and/or recrystallization is minor and CPO is weak or absent from our aggregates. Our new analyses have advantages over those from previous studies in two ways: firstly, Karato \& Lee (1999) analysed only 5-85 grains in each of four aggregates; secondly the $\mathrm{CPO} /$ dislocation density analyses conducted by Lee et al. (2002) involved two aggregates which both possessed a strong overall CPO (resulting from high shear strains).

\section{Method}

Sol-gel olivine material was prepared from dissolution of $\mathrm{Fe}$ and $\mathrm{Mg}$ nitrates in an ethanol solution (Jackson et al. 2002; Faul \& Jackson 2007). A second type of olivine material was prepared from crushed San Carlos phenocrysts (Tan et al. 2001). Cold-pressed pellets of each material were fired at $1400{ }^{\circ} \mathrm{C}$ at a $50 / 50 \mathrm{CO} / \mathrm{CO}_{2}$ gas $\operatorname{mix}$ and hotpressed in $\mathrm{Ni}_{70}-\mathrm{Fe}_{30}$ foil at $1300{ }^{\circ} \mathrm{C}$ and $300 \mathrm{MPa}$
Ar confining pressure in a Paterson gas-medium apparatus (Paterson 1990). The porosity was reduced to $1-2 \%$. After successful hot-pressing, sol-gel (H6529) and San Carlos specimens (H6694) were obtained.

Compressive deformation experiments were carried out on cylindrical specimens using the same Paterson apparatus at $300 \mathrm{MPa}$ confining pressure and $1250{ }^{\circ} \mathrm{C}$. The sol-gel sample was deformed through a series of ramps and dwells of increasing load (Faul \& Jackson 2007; run D6532, fig. 8). Care was taken to make sure (i) that steady-state creep was achieved for every dwell (visible in displacement versus time plots) and (ii) that the confining pressure was not exceeded in order to avoid embrittlement (as described by the Goetze criterion, e.g. Karato 2008). The maximum load was equivalent to a differential stress of $266 \mathrm{MPa}$ and the total strain was $18.9 \%$. The San Carlos specimen was deformed to a maximum differential stress of $227 \mathrm{MPa}$ and a total strain of $15 \%$ (run D6701). The experiments were terminated with a furnace quench to preserve the dislocation microstructure. Table 1 contains a summary of the rheological data of the deformed specimens described above.

Both specimens were sectioned perpendicular to the compressive axis, polished using colloidal silica $(0.05 \mu \mathrm{m})$ and subsequently oxidized in air at $900{ }^{\circ} \mathrm{C}$ for $45 \mathrm{~min}$ to decorate dislocations (Kohlstedt et al. 1976; Karato 1987). Subsequent polishing for $10 \mathrm{~min}$ using either $0.05 \mu \mathrm{m}$ alumina slurry (sol-gel olivine) or colloidal silica (San Carlos olivine) removed the surface oxide layer $(<1 \mu \mathrm{m}$ thick) plus $2-6 \mu \mathrm{m}$ of material to expose suitably decorated grains and dislocations. The determination of dislocation density is an extension of previous work: dislocations are decorated by oxidation (e.g. Karato et al. 1986) then imaged using backscattered electrons in a scanning electron microscope (Karato \& Lee 1999). The dislocationline-length per unit volume, a 3D dislocation density, was determined using procedures demonstrated by Farla (2010): the projected length of dislocations with a 'depth of information' value was obtained with Monte Carlo simulations of electron-olivine interactions.

A Zeiss Ultraplus field-emission scanning electron microscope (FESEM) equipped with electron 
backscatter diffraction (EBSD) capabilities, was used in this study. An accelerating voltage of $20 \mathrm{kV}$ was used with a nominal probe current of $0.6 \mathrm{nA}$. The stage was tilted to $70^{\circ}$, creating a working distance of around $25 \mathrm{~mm}$. An objective aperture of $30 \mu \mathrm{m}$ with high-current mode was used. Diffraction patterns were collected using an HKL Nordlys S Camera, equipped with a forward-scattered electron detector to provide orientation contrast (OC) images.

Manual indexing of one point per grain for solgel and San Carlos specimens was performed, guided by $\mathrm{OC}$ images. Approximate 3D dislocation density (with large uncertainty) was determined in grains to define two subsets: grains with high dislocation density $\left(\rho \sim 15 \mu \mathrm{m}^{-2}\right)$ or low dislocation density $\left(c .1 \mu \mathrm{m}^{-2}\right)$. San Carlos olivine with larger grain sizes readily allowed separation of grains into high- and low-dislocation-density classes so that no EBSD measurements were made on mediumdensity grains for this specimen. The same procedure was relatively difficult with the smaller grain size in the sol-gel aggregate.

The grain size for the San Carlos specimen was determined from an EBSD map showing 'grain' boundaries of misorientations $>10^{\circ}$, whereas the grain size of sol-gel olivine was determined from an image of decorated grain boundaries. The average grain size was calculated as the diameter of a circle of equivalent area with a sectioning bias correction of $4 / \pi$ (Jackson et al. 2002).

\section{Results}

Fourier transform infrared (FTIR) measurements confirmed the deformed sol-gel sample had a low $\mathrm{H}_{2} \mathrm{O}$ content $\left(<10\right.$ wt ppm $\mathrm{H}_{2} \mathrm{O}$, at the detection limit) and an average concentration of $26 \mathrm{wt} \mathrm{ppm}$ $\mathrm{H}_{2} \mathrm{O}$ was measured for San Carlos olivine (D6701). The measurements were carried out by taking the height of the broad band absorption around $3400 \mathrm{~cm}^{-1}$. The absorbance was calculated using the Bouguer-Beer-Lambert law (Aubaud et al. 2009) using a molar absorption coefficient of $\varepsilon=801 \mathrm{~mol}^{-1} \mathrm{~cm}^{-1}$ (Leschik et al. 2004), calibrated for water in silicate glass. No evidence of peaks was found in the absorbance spectra for structurally bound $\mathrm{OH}$ in olivine.

\section{Microstructural observations}

Figure 1 shows typical fine-grained olivine microstructures imaged using forward- and backscattered electron detectors. A heterogeneous distribution of dislocation density is evident. A binary contrast filter was applied to highlight the dislocations, which were manually recoloured in red (Fig. 1b, e).
The orientation contrast images were captured during manual indexing to facilitate separation of grains with high versus low dislocation densities. During each EBSD session, the orientation contrast images preserved the location of the analysed data points which allowed identification of the indexed grains. These are indicated by green square symbols (as shown in Fig. 1f). These grains were rediscovered using the backscatter electron detector (Fig. 1e) for further image processing and dislocation density determination. The yellow arrows indicate regions of low dislocation density, possibly resulting from grain-boundary bulging into adjacent high-dislocation-density grains (Fig. 1c, f).

\section{Pole figure analyses}

Pole figures were constructed for sol-gel and San Carlos olivine before and after the data were separated into subsets of grains of high or low dislocation density.

Figure 2a shows the $\mathrm{CPO}$ in the unseparated data for sol-gel and San Carlos olivine, one manual EBSD measurement per grain. Note that the overall patterns for preferred orientation are very weak, consistent with deformation to just 15-19\% strains. Multiples of uniform distribution (MUD) for the CPO patterns only have maximum values up to 1.75 (Fig. 2a) and should be used as a fabric strength indicator when comparing the CPO patterns from this study to others. A strong pattern will generally have a maximum MUD of 5-6. [100] lies near normal to the compression direction in either weak girdles (sol-gel) or point maxima (San Carlos), [010] is moderately concentrated subparallel to the compression direction but no pattern is identifiable for [001] (Fig. 2a). For reference, CPO from an undeformed specimen of the sol-gel material is given in Faul et al. (2011, fig. 6)

In contrast, the CPOs strengthen considerably by data separation into dislocation-density subsets (Fig. 2b, d). The CPO for the sol-gel aggregate is generally weaker than that for San Carlos olivine.

Turning first to the high-dislocation-density grains (Fig. 2b), San Carlos olivine shows a concentration of [010] oriented subparallel to the compression direction, but this is not evident in sol-gel olivine. San Carlos olivine also shows an [001] girdle normal to the compression direction while those in sol-gel olivine are possibly clustered in two separate girdles near $45^{\circ}$ to the compression direction. Comparative plots (Fig. 2c) show contours of the maximum possible Schmid factor, only for (010)[100] slip, across all grain orientations. While there is some similarity to the measured patterns in Figure 2b (e.g. San Carlos [001]), it is not strong. 

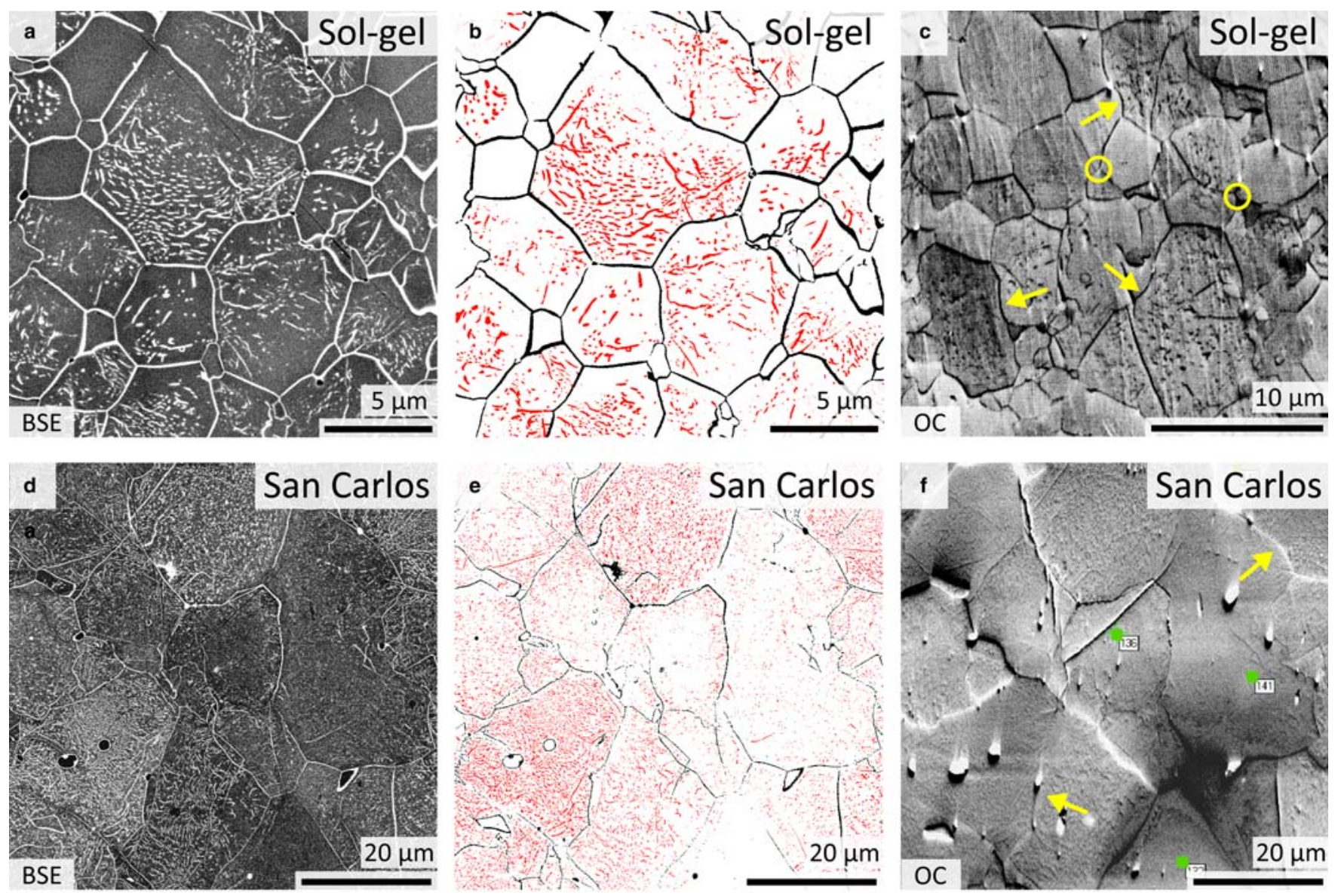

Fig. 1. (a, d) Backscattered electron images (at $5 \mathrm{kV}$ ) of sol-gel and San Carlos olivine showing grain boundaries and grain-to-grain variations of dislocation density. (b, e) Same areas but showing the processed images with dislocations highlighted in red. Images like these were used to determine the 3D dislocation density. (c) Inverted orientation contrast image (at $20 \mathrm{kV}$ ) for sol-gel olivine for another area, illustrating a similar heterogeneous distribution of dislocation densities among grains. The yellow arrows show grain-boundary bulging and the circles enclose quadruple junctions. (f) Inverted orientation contrast image (at $20 \mathrm{kV}$ ) of San Carlos olivine of the same area in panels (d) and (e) for a comparison of grains of low and high dislocation density. The green square symbols indicate grains selected for indexing. These are low-dislocation-density grains seen in panel (d). The yellow arrows indicate grain-boundary bulging into high-dislocation-density grains. 
For the low-dislocation-density grains (Fig. 2d), pole figures for [100] show concentrations perpendicular to the compression direction in both materials. CPOs for [010] and [001] are different for sol-gel and San Carlos olivine; the strongest feature is a point maximum of [001] orientations parallel to the compression direction for San Carlos, corresponding to low Schmid factor for (010)[100] slip (Fig. 2c). Note also that for San Carlos CPOs, the [010] and [001] patterns show distinct complementarity between high- versus low-dislocation-density grains.

\section{Schmid factor distributions}

Additional information can be obtained from the data through a direct comparison of calculated Schmid factor for the three main slip systems in olivine and the two dislocation-density subsets (Fig. 3). For each indexed grain, the HKL Channel software records Bunge Euler angles $\theta_{1}, \Phi$ and $\theta_{2}$ (Bunge 1982). With these Euler angles, the Schmid factor for any slip system can be determined. Histograms of the Schmid factor distribution for chosen slip systems across all grains were plotted as normalized frequencies for the high- (in red) and low(blue) dislocation-density subsets for sol-gel and San Carlos olivine (Fig. 3). Histogram data must be compared to a (green) baseline calculated for a uniform distribution of compression directions.

Three main slip systems in olivine were considered using the histogram approach, (010)[100], (001)[100] and (010)[001] (the three columns of Fig. 3). The most obvious skews exist for San Carlos olivine with (010)[100]: in Figure 3d, not only is high S clearly indicated for high-dislocationdensity grains, but the reverse is also seen for low dislocation density. For sol-gel olivine, signs of activity exist for (001)[100] (Fig. 3b) but not for (010)[100] (Fig. 3a). In contrast, Figure 3a, c, e generally show trends that are indistinguishable from the uniform-distribution baseline. Figure $3 f$ is different again; the distribution is skewed towards high $\mathrm{S}$ for low-dislocation-density grains and conflicts with that of the corresponding high-density grains where the trend is indistinguishable from uniform. (010)[001] slip is therefore not indicated for either olivine aggregate by this type of analysis.

\section{Comparison of Schmid factor and dislocation density in inverse pole figures}

Where pole figures show how crystallographic directions of grains are distributed in the reference frame of the sample, inverse pole figures (IPFs) show how a chosen direction in the sample reference frame is distributed in the reference frame of the crystal. In Figure 4 the compression direction on each grain is plotted as an IPF for both high and low-dislocation-density subsets. In addition, Figure $4 \mathrm{c}$ shows (red regions in the same IPF space) orientations for compression directions that would produce high Schmid factors $(>0.4)$ on each of the three slip systems already used in the previous section. The compression directions that yield maximum Schmid factor of 0.5 on single slip systems are indicated as $[\mathrm{hkl}]_{\mathrm{c}}$ where subscript c indicates the direction is in cartesian (not crystallographic) space; [101] $]_{\mathrm{c}}$ bisects [100] and [001] directions. No strong similarity to the measured IPFs is obvious for any one of the three slip systems chosen.

In IPF representation, San Carlos olivine shows a CPO somewhat stronger (note maximum MUD between 2 and 3) than for sol-gel olivine. For both, there is distinct complementarity between low- and high-dislocation-density grains. Few grains of high dislocation density have [001] axes parallel to the compression direction, orientations with very low Schmid-factors for all of the slip systems.

In San Carlos olivine, compression directions for high-dislocation-density grains lie mainly from [010] to $[110]_{\mathrm{c}}$, which partially corresponds to high Schmid factor values on (010)[100], (noting though that grains with a compression direction near [010] had low Schmid factors for all three slip systems). For low-dislocation-density grains in San Carlos, compression directions cluster between [001] and $[011]_{c}$, the direction for maximum Schmid factor on (010)[001].

Sol-gel olivine shows a different pattern. In high-dislocation-density grains, many compression axes are near [101] $]_{\mathrm{c}}$ (possible (001)[100] slip) while few compression axes have high Schmid-factor values for (010)[100] slip. For low-dislocation-density grains, the most dominant clustering occurs near high Schmid factors for either (010)[100] or (010)[001] slip systems.

\section{Rotation on sub-boundaries}

Lattice rotation (misorientation) axes across subgrain boundaries $\left(2-10^{\circ}\right)$ were obtained from EBSD map data (many measurements per grain) of the San Carlos specimen (Fig. 5) only. The data is represented in a crystal frame similar to the inverse pole figure above, but Figure 5 instead shows misorientation-axis distribution. Misorientation axes dominantly lie near [001] with others near [010].

\section{Discussion}

\section{The effect of rheology on slip-system activation}

Compressive deformation to modest strains in this study led to the overall development of very weak 


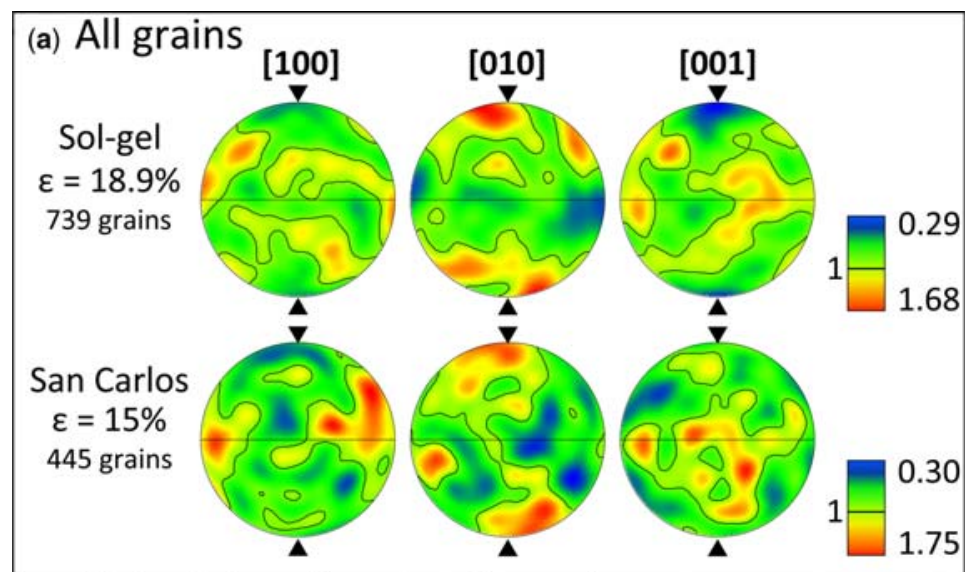

(b) High-dislocation-density grains

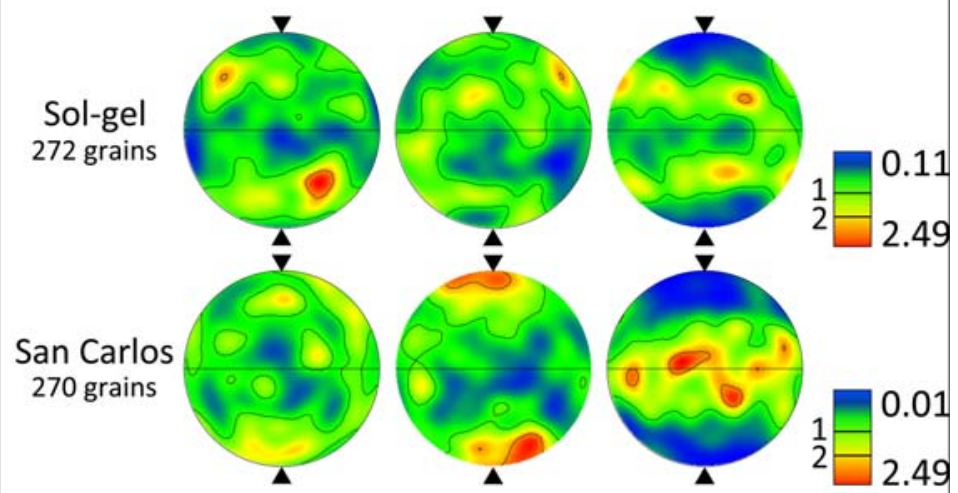

(c) Maximum Schmid factor for (010)[100]

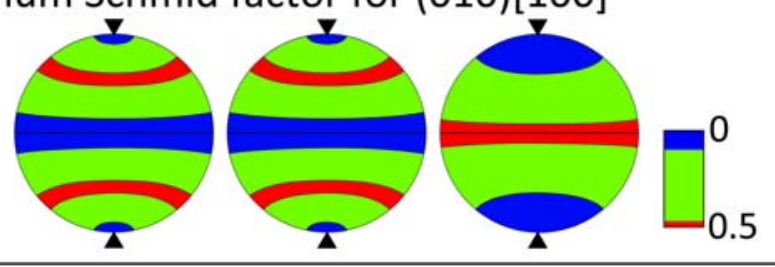

(d) Low-dislocation-density grains

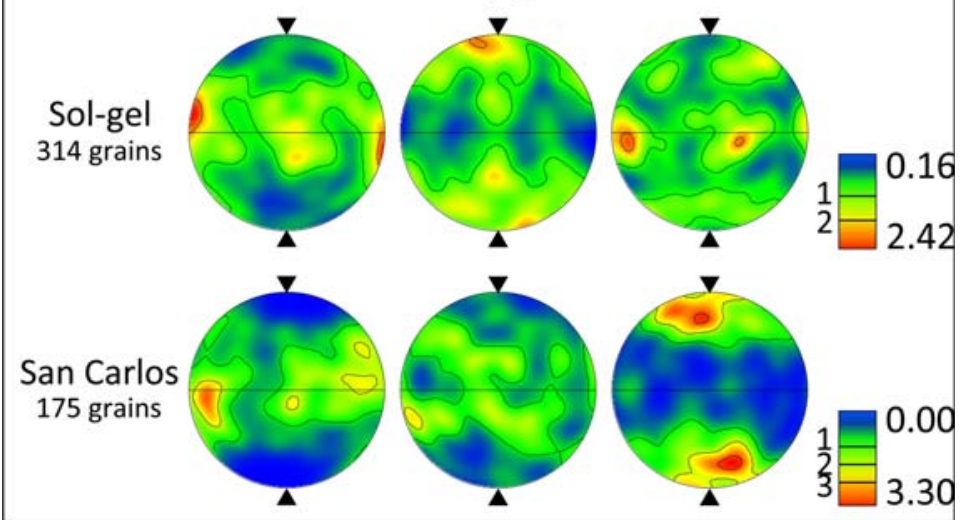

Fig. 2. 
CPO in sol-gel and San Carlos olivine with maximum MUD values of only 1.75 , stronger for San Carlos olivine (Fig. 2a). This result is likely influenced by many parameters such as grain size, impurity content, water content and possibly melt fraction, the latter three essentially absent from sol-gel olivine. The strain rate/stress data for sol-gel olivine are presented in Faul et al. (2011) and for San Carlos olivine in Farla (2010). During deformation in the diffusion creep regime, both specimens demonstrated similar strain rates $\left(5 \times 10^{-6} \mathrm{~s}^{-1}\right.$ at $\left.110 \mathrm{MPa}\right)$ despite the difference in grain size (see Table 1). In the dislocation creep regime, San Carlos olivine was significantly weaker than sol-gel olivine.

\section{Slip-system analysis}

By separating high- and low-dislocation-density grains, we attempt to correlate Schmid factor with dislocation density in both olivine specimens. The grain orientations of both groups of high- and low-dislocation-density grains show weak CPO patterns and, quite noticeably, some complementarity of the CPO between the two groups. Complementarity is most pronounced in San Carlos for the [001] direction (Fig. 2b, c). Such an observation can be used to infer that grains in favourable orientations deformed via 'easy' slip and developed high dislocation densities. On the other hand, grains in unfavourable orientations for slip presumably deformed little and should have low dislocation densities.

If this inference is true, then a signature of (010)[100] slip (the easiest system in olivine) should be observable in the CPO for grains of high dislocation density. As the schematic pole figures in Figure 2c demonstrate, the CPO of grains with high (010)[100] Schmid factor should feature two girdles for [100] and [010] at $45^{\circ}$ from the compression direction; such girdles possibly exist for sol-gel olivine but certainly not for San Carlos olivine (Fig. 2). In addition, a girdle normal to the compression direction should occur for [001]. A pronounced feature of this type is seen for San Carlos olivine, but not for sol-gel olivine
(Fig. 2b). Complementary patterns should be observed at low dislocation density given that the overall CPO is essentially uniform (see Fig. 2a). Parallel to the compression direction at low dislocation density (Fig. 2d), San Carlos grains show distinct [001] concentration whereas sol-gel olivine shows a weaker [010] maximum.

Evidence for activation of a slip system can possibly be deduced more readily by skews in the histograms of Figure 3 relative to the uniform distribution if reverse trends exist for high- versus low-dislocation-density grains. On this basis, there is evidence for activation of (010)[100] in San Carlos olivine (Fig. 3d) and (001)[100] in sol-gel olivine (Fig. 3b). Both low- and high-dislocationdensity subsets are indistinguishable from the green baseline in three cases (Fig 3a, c, e). San Carlos olivine even shows some trend against activation of (010)[001] (Fig. 3f). The histograms show no single slip system to have been dominant in either specimen.

In the IPFs of Figure 4, concentrations of the compression direction for high-dislocation-density grains are expected to lie near $[110]_{\mathrm{c}}$ for (010)[100] slip, near [101] for (001)[100] slip and near $[011]_{c}$ for (010)[001] slip (Fig. 4c). Neither specimen clearly shows a pattern suggestive of single slip. However sol-gel olivine at high dislocation density does show clustering in the vicinity of $[101]_{c}$, suggesting that (001)[100] has been active. San Carlos, on the other hand, appears to have deformed on (010)[100], although the highest concentration of the compression direction in high-dislocation-density grains lies near to [010]. Typically, this is observed when the grains are able to rotate during plastic deformation (Karato 2008). However, given the low strain in our experiments, multiple slip systems and grain-to-grain stress heterogeneities are more likely explanations.

Misorientation-axis data $\left(2-10^{\circ}\right.$ rotations $)$ plotted in Figure 5 can be compared with those expected for the three main slip systems and two types of dislocation substructures, tilt versus twist boundaries. For a single slip system (i.e. tilt boundaries of edge dislocations), [001] misorientations measured on internal boundaries strongly indicate

Fig. 2. Pole figures presenting grain orientation data for both sol-gel and San Carlos olivine. The compression direction is represented by pairs of black arrows. The half-scatter width is $20^{\circ}$ with $5^{\circ}$ clustering. An equal-area lower-hemisphere projection was used. (a) Pole figures for all measured grains. The patterns are weak with low maximum multiples of uniform distribution (MUD) values (see legends). Concerning the number of grains analysed, for sol-gel olivine it includes grains of medium dislocation density but, for San Carlos olivine, it excludes them. (b) and (d) show pole figures separated into grain subsets of high or low dislocation density; note that higher maximum MUD values here indicate stronger preferred orientation. The CPO patterns in the pole figures between high and low dislocation density partially complement each other for both sol-gel and San Carlos olivine. (c) Pole figures contoured for the range of maximum possible Schmid factors on slip system (010)[100] plotted for all grain orientations (also equal-area projections). 

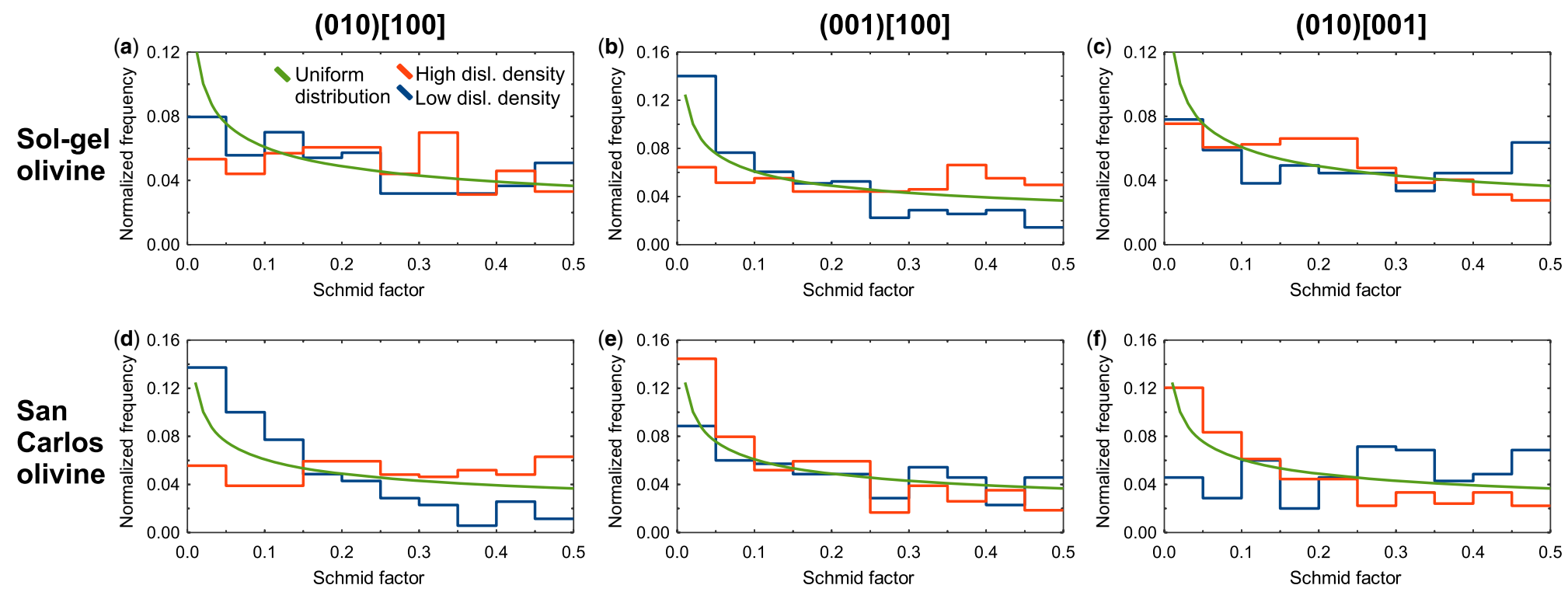

Fig. 3. Schmid-factor distributions for sol-gel and San Carlos olivine for the three main slip systems (010)[100], (001)[100] and (010)[001]. The frequency of grains ( $y$-axis) has been normalized for comparison. The subsets of high- and low-dislocation-density grains are shown by the red and blue lines, respectively, presented as histograms at 0.05 Schmid factor intervals. As a reference, the green curve shows a baseline calculation of normalized frequency for Schmid factors of a uniform distribution of grain orientations.

Significant deviations from the green curve could indicate activation of a slip system. 


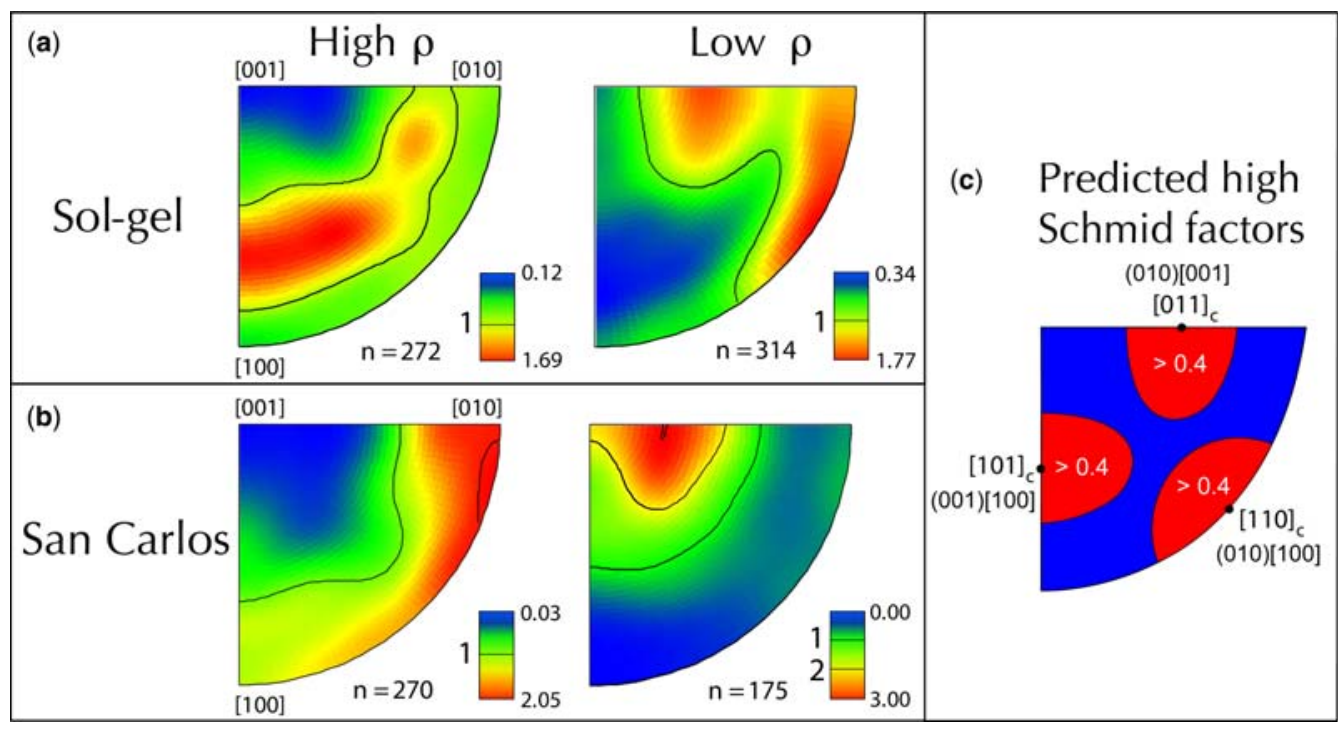

Fig. 4. (a, b) Equal-area inverse pole figures in which the compression direction is plotted in the olivine crystal frame for sol-gel and San Carlos olivine of both dislocation density subsets. Projections are equal-area with a half-scatter width of $20^{\circ}$ and $5^{\circ}$ clustering. Also, $\mathrm{n}$ is the number of grains analysed. (c) An additional inverse pole figure shows orientations of compression axes for the highest Schmid factors $(>0.4)$ on the three main slip systems in olivine.

slip in San Carlos olivine grains on (010)[100], but also minor contribution from (001)[100] required to explain the [010] rotations. Alternatively, a twistboundary geometry involving two families of screw dislocations with Burgers vectors either [100] or

\section{2-10 rotations}

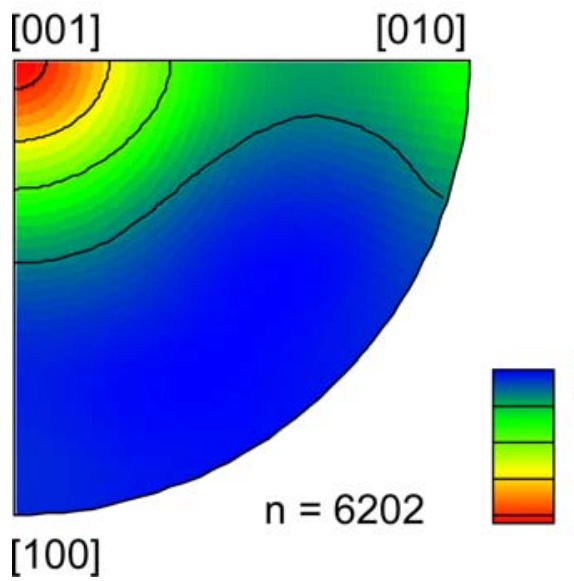

0.38

Fig. 5. Plot of misorientation-axis data for low angle $\left(2-10^{\circ}\right)$ internal sub-grain boundaries shown in the crystallographic reference frame; data computed from the San Carlos EBSD map. The half-scatter width is $20^{\circ}$ and $3^{\circ}$ clustering. Here, $n$ is the number of points (not grains) analysed.
[001] would explain this [010] rotation axis. These deductions are broadly consistent with results in Figures 3 and 4.

Discrepancies between Schmid factor and dislocation density in Figures 2b, d, 3 and 4 suggest that a model for uniform stress (Sachs) cannot be valid. Put a different way, slip in high-Schmid-factor grains could possibly have been suppressed by stress transfer to surrounding 'hard' grains. In addition, stress heterogeneity is supported by considerable grain-to-grain variations in dislocation density and by weak patterns from the measured pole figures and IPFs. Note that conclusions about stress heterogeneity were similarly reached by Karato \& Lee (1999) and Lee et al. (2002).

Furthermore, there is evidence for operation of multiple slip systems in our deformed aggregates. For San Carlos olivine, we conclude that (010)[100] operated during deformation (Figs 3d $\& 4 b$ and especially Fig. 5), but all the analyses suggest some contributions from other slip systems. For sol-gel olivine, (001)[100] is apparently active (Figs 3b \& 4a) but again accompanied by other slip systems.

\section{Comparison with previous studies}

The dislocation-density distribution and substructures that evolve during deformation of our fine-grained olivine appear to be different than in olivine aggregates undergoing dynamic 
recrystallization under high-strain simple shear (Karato \& Lee 1999; Lee et al. 2002). Commonly, a heterogeneous distribution of dislocation density is observed in low-OH deformed olivine polycrystals. This may result from varying intra-granular stresses between grains, where grains which are favourably oriented are more likely to deform. However, from high-strain deformation experiments, such grains are less likely to dominate the final preferred orientations caused by grainboundary migration (Zhang et al. 2000).

In this study, some evidence for grain-boundary bulging and sliding between grains is observed, most clearly for fine-grained sol-gel olivine (see Fig. 1) but not at the expense of a significant increase in grain-boundary area or an increase in low-dislocation-density grains. Grain shapes are not typical of high-temperature stress-induced grain-boundary migration and vary between equigranular polygonal or mild interlobate structures (Passchier \& Trouw 1998).

It is not well known to what degree the microstructural state of stress and strain plays a role between grains (Taylor 1938; Karato \& Lee 1999). However, a second-order-estimate model has recently (Castelnau et al. 2008) simulated stress heterogeneities in polycrystalline olivine. This model essentially falls between the Taylor (uniform strain) and Sachs (uniform stress) end-member models. The second-order modelling highlights the likelihood of localization of stress and strain in a deforming olivine polycrystal with local stresses and strains significantly higher or lower than the imposed (bulk) macroscopic equivalents. All the grains in the model of Castelnau et al. (2008) were allowed five degrees of freedom via the three known slip systems in olivine plus two 'arbitrarily' assigned systems. While the details of the chosen parameters are debatable, the effects of strain localization, fluctuations and stress gradients in and between grains have important consequences for microstructural evolution in olivine aggregates.

\section{Conclusion}

This study for weakly strained (c. 15-19\%) synthetic olivine aggregates shows a near-uniform $\mathrm{CPO}$, together with low maximum-MUD values. An overall near-uniform CPO offered a good possibility for identification of operating slip systems in olivine, partly through searching for complementarity expected between grain populations of high and low dislocation density without significant impacts from grain rotation or recrystallization during low-strain deformation.

Patterns of preferred orientation from pointindexed grains of high and low dislocation density, of Schmid-factor distribution and of misorientation-axis data arising from an EBSD map have been analysed for evidence of correlation between Schmid factor (on three olivine slip systems) and dislocation density. The patterns show many complexities that defy explanation by activation of any one slip system, although the misorientation-axis analysis (San Carlos olivine only) does appear to allow a straightforward interpretation. For coarser-grained San Carlos olivine, there is strong evidence from all analyses that grains of high dislocation density are favourably oriented to deform via the slip system (010)[100], but probably with important contributions from other slip systems. For sol-gel olivine, equivalent analyses suggest (001)[100] slip but the evidence is weaker than for San Carlos olivine and also suggestive of contributions from multiple slip systems. Perhaps differences in grain size, $\mathrm{OH}$ and impurity content could have played a role in the slip-system contrast between these two types of olivine aggregate, although more experimental evidence is needed to confirm the patterns.

Heterogeneous stress provides an explanation for deformation of grains in unfavourable orientations relative to the external compressive stress (i.e. with low Schmid factor). Variation in dislocation density between grains indicates that strain is heterogeneous. Furthermore, the CPO data described above re-affirm that neither the Sachs model of uniform stress nor the Taylor model of uniform strain are adequate to describe plastic deformation and textural evolution of olivine. Plastic deformation involves multiple slip systems and is more comparable to advanced simulations such as the second-order micromechanical model of Castelnau et al. (2008).

We thank F. Brink for his assistance with FESEMs and EBSD at the ANU Centre for Advanced Microscopy in the Australian Microscopy \& Microanalysis Research Facility. R. Farla acknowledges the award of an Endeavour International Postgraduate Scholarship by the Australian Government.

\section{References}

Aubaud, C., Bureau, H., Raepsaet, C., Khodja, H., Withers, A. C., Hirschmann, M. M. \& Bell, D. R. 2009. Calibration of the infrared molar absorption coefficients for $\mathrm{H}$ in olivine, clinopyroxene and rhyolitic glass by elastic recoil detection analysis. Chemical Geology, 262, 78-86.

Ben Ismaïl, W. \& MainPrice, D. 1998. An olivine fabric database: an overview of upper mantle fabrics and seismic anisotropy. Tectonophysics, 296, $145-157$.

Bunge, H. J. 1982. Texture Analysis in Materials Science: Mathematical Models. Butterworths, London. 
Castelnau, O., Blackman, D. K., Lebensohn, R. A. \& Ponte Castaneda, P. 2008. Micromechanical modeling of the viscoplastic behaviour of olivine. Journal of Geophysical Research, 113, B09202.

Dawson, P. R. \& WenK, H. R. 2000. Texturing of the upper mantle during convection. Philosophical Magazine A, 80, 573-598.

Drury, M. R. \& PenNock, G. M. 2007. Subgrain rotation recrystallization in minerals. In: Prangnell, P. B. \& BAte, P. S. (eds) Materials Science Forum, Fundamentals of Deformation and Annealing. Trans Tech Publications, Switzerland, 550, 95-104.

FARLA, R. J. M. 2010. An exploratory study of dislocation relaxation in polycrystalline olivine. $\mathrm{PhD}$ thesis, The Australian National University.

FAUL, U. H. \& JACKSON, I. 2007. Diffusion creep of dry, melt-free olivine. Journal of Geophysical Research, 112, $1-14$.

Faul, U. H., Fitz Gerald, J. D., Farla, R. J. M., AhleFELDT, R. \& JACKSON, I. 2011. Dislocation creep of fine-grained olivine. Journal of Geophysical Research, 116. http://dx.doi.org/10.1029/2009JB007174.

Goetze, C. 1978. Mechanisms of creep in olivine. Philosophical Transactions of the Royal Society London, 288, 99-119.

Green, H. W. \& Radcliffe, S. V. 1972. Dislocation mechanisms in olivine and flow in upper mantle. Earth and Planetary Science Letters, 15, 239.

Hull, D. \& BACON, D. J. 2001. Introduction to Dislocations. 4th edn. Butterworth-Heinemann, Oxford, UK.

Jackson, I., Fitz Gerald, J. D., Faul, U. H. \& Tan, B. H. 2002. Grain-size sensitive seismic wave attenuation in polycrystalline olivine. Journal of Geophysical Research B, 107, 2360 .

JunG, H. \& Karato, S. 2001. Water-induced fabric transitions in olivine. Science, 293, 1460-1463.

Jung, H., Katayama, I., Jiang, Z., Hiraga, T. \& Karato, S. 2006. Effect of water and stress on the latticepreferred orientation of olivine. Tectonophysics, 421, $1-22$.

KARATO, S. 1987. Scanning electron microscope observation of dislocations in olivine. Physics and Chemistry of Minerals, 14, 245-248.

KaRATO, S. 1988. The role of recrystallization in the preferred orientation of olivine. Physics of the Earth and Planetary Interiors, 51, 107-122.

Karato, S. 2008. Deformation of Earth Materials: An Introduction to the Rheology of Solid Earth. Cambridge University Press, Cambridge.

Karato, S. \& LEE, K. H. 1999. Stress-strain distribution in deformed olivine aggregates: inference from microstructural observations and implications for texture development. In: Proceedings of the Twelfth International Conference on Textures of Materials, Montréal, Canada, 1546-1555.

Karato, S. I., Paterson, M. S. \& Fitz Gerald, J. D. 1986. Rheology of synthetic olivine aggregates- influence of grain-size and water. Journal of Geophysical Research B, 91, 8151-8176.

Kohlstedt, D. L., Goetze, C., Durham, W. B. \& VANDERSANDE, J. 1976. New technique for decorating dislocations in olivine. Science, 191, 1045-1046.

Lee, K., JiAng, Z. \& Karato, S. 2002. A scanning electron microscope study of the effects of dynamic recrystallization on lattice preferred orientation in olivine. Tectonophysics, 351, 331-341.

LeschiK, M., HeIDE, G. ET AL. 2004. Determination of $\mathrm{HO}$ and DO contents in rhyolitic glasses. Physics and Chemistry of Glasses, 45, 238-251.

Lister, G. S., Paterson, M. S. \& HobBs, B. E. 1978. The simulation of fabric development in plastic deformation and its application to quartzite: the model. Tectonophysics, 45, 107-158.

Passchier, C. W. \& Trouw, R. A. J. 1998. Microtectonics. 2nd edn. Springer, Berlin Heidelberg, Germany.

Paterson, M. S. 1990. Rock deformation experimentation. In: DubA, A. G. (ed.) The Brittle-Ductile Transition in Rocks. American Geophysical Union, Washington, Geophysical Monograph Series, 56, 187-194.

Ribe, N. M. \& Yu, Y. 1991. A theory for plastic deformation and textural evolution of olivine polycrystals. Journal of Geophysical Research B, 96, 8325-8335.

TAN, B. H., JACKSON, I. \& Fitz Gerald, J. D. 2001. Hightemperature viscoelasticity of fine-grained polycrystalline olivine. Physics and Chemistry of Minerals, 28, 641-664.

TAYLOR, G. I. 1938. Plastic strain in metals. Journal of the Institute of Metals, 62, 307-324.

Tommasi, A., Mainprice, D., Canova, G. \& Chastel, Y. 2000. Viscoplastic self-consistent and equilibriumbased modeling of olivine lattice preferred orientations: implications for the upper mantle seismic anisotropy. Journal of Geophysical Research, 105 , 7893-7908.

VON MisEs, W. 1928. Mechanik der plastischen formanderung von kristallen. Applied Mathematics and Mechanics, $\mathbf{8}, 161$.

Wenk, H. R., Bennett, K., Canova, G. R. \& Molinari, A. 1991. Modelling plastic deformation of peridotite with the self-consistent theory. Journal of Geophysical Research B, 96, 8337-8349.

White, S. H. 1979. Grain and sub-grain size variations across a mylonite zone. Contributions to Mineralogy and Petrology, 70, 193-202.

Winther, G., Jensen, D. J. \& Hansen, N. 1997. Modelling flow stress anisotropy caused by deformation induced dislocation boundaries. Acta Materialia, 45, 2455-2465.

Zhang, S. Q., Karato, S., FitzGerald, J. D., Faul, U. H. \& Zhou, Y. 2000. Simple shear deformation of olivine aggregates. Tectonophysics, 316, 133-152. 